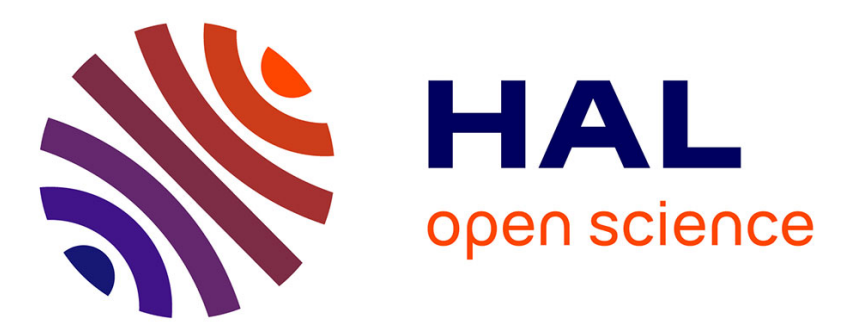

\title{
A contribution to the discovery of multidimensional patterns in healthcare trajectories
} \author{
Elias Egho, Nicolas Jay, Chedy Raïssi, Dino Ienco, Pascal Poncelet,
} Maguelonne Teisseire, Amedeo Napoli

\section{> To cite this version:}

Elias Egho, Nicolas Jay, Chedy Raïssi, Dino Ienco, Pascal Poncelet, et al.. A contribution to the discovery of multidimensional patterns in healthcare trajectories. Journal of Intelligent Information Systems, 2014, 42 (2), pp.283 - 305. 10.1007/s10844-014-0309-4 . hal-01094377

\section{HAL Id: hal-01094377 \\ https://inria.hal.science/hal-01094377}

Submitted on 13 Jan 2015

HAL is a multi-disciplinary open access archive for the deposit and dissemination of scientific research documents, whether they are published or not. The documents may come from teaching and research institutions in France or abroad, or from public or private research centers.
L'archive ouverte pluridisciplinaire HAL, est destinée au dépôt et à la diffusion de documents scientifiques de niveau recherche, publiés ou non, émanant des établissements d'enseignement et de recherche français ou étrangers, des laboratoires publics ou privés. 


\title{
A contribution to the discovery of multidimensional patterns in healthcare trajectories
}

\author{
Elias Egho ${ }^{1}$, Nicolas Jay ${ }^{1}$, Chedy Raïssi ${ }^{1}$, Dino Ienco $^{2}$, Pascal Poncelet ${ }^{2}$, \\ Maguelonne Teisseire ${ }^{2}$ and Amedeo Napoli ${ }^{1}$ \\ 1 LORIA (CNRS - Université de Lorraine) / Inria Nancy Grand Est \\ \{elias.egho, nicolas.jay, amedeo.napoli\}@loria.fr \\ \{chedy.raissi\}@inria.fr \\ 2 Irstea, UMR TETIS / LIRMM, Univ. Montpellier 2 \\ $\{$ dino.ienco, maguelonne.teisseire\}@teledetection.fr \\ \{pascal.poncelet\}@lirmm.fr
}

\begin{abstract}
Sequential pattern mining is aimed at extracting correlations among temporal data. Many different methods were proposed to either enumerate sequences of set valued data (i.e., itemsets) or sequences containing dimensional items. However, in real-world scenarios, data sequences are described as combination of both multidimensional items and itemsets. These heterogeneous descriptions cannot be handled by traditional approaches. In this paper we propose a new approach called MMISP (Mining Multidimensional Itemset Sequential Patterns) to extract patterns from complex sequential database including both multidimensional items and itemsets. The novelties of the proposal lies in: (i) the way in which the data are efficiently compressed; (ii) the ability to reuse and adopt sequential pattern mining algorithms and (iii) the extraction of new kind of patterns. We introduce a case-study on real-world data from a regional healthcare system and we point out the usefulness of the extracted patterns. Additional experiments on synthetic data highlights the efficiency and scalability of the approach MMISP.
\end{abstract}

Keywords: complex sequential patterns, multidimensional sequential patterns, data mining, complex data

\section{Introduction}

Real-world databases can be viewed as large and complex sources of information that can be analyzed for discovering new knowledge units or for decision making [15]. When the temporal dimension is also considered, every bit of information or event is associated with a timeline describing a total order over events. This total ordering introduces complexity in the extraction process. Many efficient approaches were developed to mine patterns depending on time or order, such as PrefixSpan [7], SPADE [16], ClosSpan [12],...etc. These approaches focus on a single dimensional sequence dataset. However, there are many situations in 
which a database can be multidimensional, i.e. several characteristics of data can be ordered over time. Pinto et al. [8], Zhang et al. [17] and Yu et al. [14] introduced the notion of multidimensionality in a sequence and proposed several algorithms to mine this type of data. In data warehouse environments, a background knowledge is usually available in form of taxonomies, classification or concept hierarchies. Based on that, Plantevit et al. introduced $M^{3} S P$ [9], an algorithm able to incorporate several dimensions and the possible associated posets within the sequential pattern mining process.

The above approaches focus on sequences of homogeneous items. They do not pay attention to real-world complex data described by a vector of heterogeneous elements with different types, i.e. item or itemset. For example, in the healthcare domain, a patient trajectory is defined as a sequence of hospitalizations, where each hospitalization is defined as a vector of three heterogeneous elements: (i) healthcare institution, (ii) diagnosis and (iii) set of medical procedures. The healthcare institution and the diagnosis can be encoded as variables taking values, where values are organized within posets, while medical procedures are not comparable. This example shows that each dimension in data has to be managed in a proper and suitable way.

In this paper, we present an approach for mining multidimensional and heterogeneous patterns from medical patient trajectories, i.e. the trajectory of a patient visiting several hospitals. Our objective is to discover interesting patterns able to characterize patient stays and associated medical procedures. Such patterns can be interpreted by healthcare professionals to better understand patient pathways and improve the organization of care. Such multidimensional and heterogeneous patterns have to be mined by adapted and suitable methods. Accordingly, in this paper, we propose a new method to extract sequential patterns from databases including sequences of heterogeneous vectors. In addition, the approach is able to take into account background knowledge lying in term posets. The approach is original and efficient. An adapted algorithms is proposed which shows a very good behaviour on real-world medical data.

The remainder of this paper is organized as follows, Section 2 describes related work in classical and multidimensional sequential patterns. Section 3 introduces the problem statement and a running example. The algorithm for extracting complex frequent patterns is described in Section 4. Section 5 presents experimental results from both quantitative and qualitative points of views and Section 6 concludes the paper.

\section{Related Work}

Agrawal and Srikant [1] introduced the problem of mining sequential patterns over large sequential databases. Formally, given a set of sequences, where each sequence is a list of transactions ordered by time and each transaction is a set of items, the problem amounts to find all frequent subsequences that appear a sufficient number of times with a user-specified minimum support threshold (minsup). Following the work of Agrawal and Srikant many studies have con- 
tributed to the efficient mining of sequential patterns [6]. Most of them are based on the Apriori property, which states that any super pattern of a non-frequent pattern cannot be frequent. The main algorithms are PrefixSpan [7], SPADE [16], SPAM [2], PSP [5], DISC [3], PAID [13] and FAST [10]. All these algorithms aim at discovering sequential patterns from a set of sequences of itemsets such as customers who frequently buy DVDs of episodes I, II and III of Stars Wars, then buy within 6 months episodes IV, V, VI of the same famous epic space opera.

Many studies about sequential patterns discovery focus on single-dimensional sequences. However, in many situations, the database is multidimensional in the sense that items can be of different nature. For example, a consumer database can hold information such as article price, gender of the customer, location of the store and so on. Pinto et al [8] proposed the first work for mining multidimensional sequential patterns. In this work, a multidimensional sequential database is defined as a schema $\left(I D, D_{1}, \ldots, D_{m}, S\right)$, where $I D$ is a unique customer identifier, $D_{1}, \ldots, D_{m}$ are dimensions describing the data and $\mathrm{S}$ is the sequence of itemsets. A multidimensional sequence is defined as a vector $\left\langle\left\{d_{1}, d_{2}, \ldots, d_{m}\right\}, S_{1}, S_{2}, \ldots, S_{l}\right\rangle$ where $d_{i} \in D_{i}$ for $(i \leqslant m)$ and $S_{1}, S_{2}, \ldots, S_{l}$, are the itemsets of sequence $S$. For instance, $\left\langle\{\right.$ Paris, Male $\left.\},\left\{m p_{1}, m p_{2}\right\},\left\{m p_{3}\right\}\right\rangle$ describes a male patient who underwent procedures $m p_{1}$ and $m p_{2}$ in Paris and then underwent $m p_{3}$ also in Paris. Here, dimensions remain constant over time, such as the location of the treatment. This means that it is not possible to have a pattern indicating that when the patient underwent procedures $m p_{1}$ and $m p_{2}$ in Paris then he underwent $m p_{3}$ in Nancy. Among other proposals, Yu et al [14] proposed two methods AprioriMD and PrefixMDSpan for mining multidimensional sequential patterns in the web domain. This study considers pages, sessions and days as dimensions. Actually, these three different dimensions can be projected into a single dimension corresponding to web pages, gathering web pages visited during a same session and ordering sessions w.r.t the day as order.

In real world applications, each dimension can be represented at different levels of granularity, by using a poset. For example, apples in a market basket analysis can be either described as fruits, fresh food or food. The interest lies in the capacity of extracting more or less general/specific multidimensional sequential patterns and overcome problems of excessive granularity and low support. Srikant and Agrawal [11] proposed GSP which uses posets for extracting sequential patterns. The basic approach is based on replacing every item with all the ancestors in the poset and then the frequent sequences are generated. This approach is not scalable in a multidimensional context because the size of the database becomes the product of maximum height of the posets and number of dimensions. Plantevit et al [9] defined a multidimensional sequence as an ordered list of multidimensional items, where a multidimensional item is a tuple $\left(d_{1}, \ldots, d_{m}\right)$ where $d_{i}$ is an item associated with the $i^{\text {th }}$ dimension. They proposed $M^{3} S P$, an approach taking both aspects into account where each dimension is represented at different levels of granularity, by using a poset. $M^{3} S P$ is able to search for sequential patterns with the most appropriate level 
of granularity. Their approach is based on the extraction of the most specific frequent multidimensional items, which are then used as alphabet to rephrase the original database. Then, $M^{3} S P$ uses a standard sequential pattern mining algorithm to extract multidimensional sequential patterns. However, $M^{3} S P$ is not adapted to mine sequential databases, where sequences are defined over a combination of sets of items and items lying in a poset. Then it is not possible to have a pattern indicating that when the patient went to $u h_{p}$ for a problem of cancer $c a$, where he underwent procedures $m p_{1}$ and $m p_{2}$, then he went to $g h_{l}$ for the same medical problem $c a$, where he underwent $m p_{3}$ (i.e, $\left.\left\langle\left(u h_{p}, c a,\left\{m p_{1}, m p_{2}\right\}\right),\left(g h_{l}, c a,\left\{m p_{3}\right\}\right)\right\rangle\right)$.

Compared to $M^{3} S P$, the main contribution of this article is to generalize the concept of multidimensional sequence by considering multidimensional itemsets instead of multidimensional items. In our approach, an event in a sequence can be seen as a vector of itemsets, whenever $M^{3} S P$ represents an event as a tuple of atomic elements. This restriction prevents $M^{3} S P$ from extracting condensed and heterogeneous patterns.

\section{Problem Statement}

\subsection{An introductory example}

Firstly we propose an example to illustrate the present approach. The French healthcare system called PMSI ${ }^{3}$ is a national information system used in France to manage hospital activity with both an economical and a medical points of view [4]. In this system, each patient's stay is a standardized record of administrative and clinical data. Accordingly, each hospitalization can be formalized along three dimensions: $(i)$ healthcare institution, $(i i)$ diagnosis and (iii) medical procedures. The first two dimensions, i.e. healthcare institutions and diagnosis, are considered as variables whose values are lying in a poset. The last dimension is about medical procedures and can be considered as a variable which is setvalued. The basic sets of the healthcare institutions $H$, the diagnosis DG and the medical procedures MP, are the following:

- $\mathrm{H}=\left\{T_{h}, u h, g h, u h_{p}, u h_{n}, g h_{p}, g h_{l}\right\}$.

- DG $=\left\{T_{d}, c a, r, r_{1}, r_{2}, c a_{1}, c a_{2}, c a_{3}\right\}$.

- MP $=\left\{m p_{1}, m p_{2}, m p_{3}, m p_{4}\right\}$.

Hospital and diagnosis can be described at different levels of granularity through two posets $(H, \leqslant)$ and $(D G, \leqslant)$ which are defined in Figure 1.

In the poset $(H, \leqslant), u h_{p}$ and $u h_{n}$ denote university hospitals, $u h$, with $u h_{p} \leqslant$ $u h$ and $u h_{n} \leqslant u h ; g h_{p}$ and $g h_{l}$ are general hospitals, $g h$, with $g h_{p} \leqslant g h$ and $g h_{l} \leqslant g h$.

The hospitalization of a patient is defined as a vector with three components, $(h, d g, m p)$. The component $h$ refers to institution and its value lies in the

\footnotetext{
${ }^{3}$ Programme de Médicalisation des Sytèmes d'Information.
} 


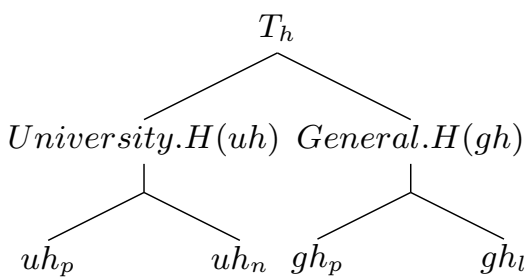

Healthcare Institution $\left(H, \leqslant_{h}\right)$

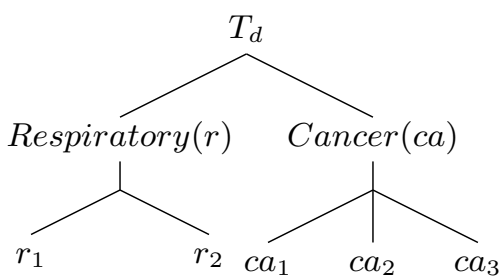

Diagnosis $(D G, \leqslant d g)$

Fig. 1: The posets for the healthcare institution set $\mathrm{H}$ and the diagnosis set DG

partially ordered set $(H, \leqslant)$. The same thing applies to $d g$ with $(D G, \leqslant)$. The component $m p$ refers to medical procedures and is a set-valued.

Then, $\left(u h_{p}, c a_{1},\left\{m p_{1}, m p_{2}\right\}\right)$ describes an hospitalisation, where $u h_{p} \in H, c a_{1} \in$ $D G$ and $\left\{m p_{1}, m p_{2}\right\} \subseteq M P$.

\subsection{Basic definitions}

Definition 1. (Elementary sequence)

An elementary sequence $e=\left(e_{1}, e_{2}, \ldots, e_{n}\right)$ is defined as a vector of $n$ elements where an element can be either:

- an atomic element taken from a partially order set.

- a subset of a non ordered set.

Definition 2. (Ordering of elementary sequences)

Given two elementary sequences $e=\left(e_{1}, e_{2}, \ldots, e_{n}\right)$ and $e^{\prime}=\left(e_{1}^{\prime}, e_{2}^{\prime}, \ldots, e_{n}^{\prime}\right), e$ is more specific than $e^{\prime}$, denoted by $e \leq_{e} e^{\prime}$, if for all $i=1 \ldots n$ we have:

$e_{i} \leqslant e_{i}^{\prime} \quad$ where $e_{i}, e_{i}^{\prime}$ are elements of a poset.

$e_{i}^{\prime} \subseteq e_{i} \quad$ where $e_{i}, e_{i}^{\prime}$ are sets.

Example 1. $e=\left(u h_{p}, c a_{1},\left\{m p_{1}, m p_{2}\right\}\right)$ is an elementary sequence more specific than $e^{\prime}=\left(u h, T_{d},\left\{m p_{1}\right\}\right)$ as:

$-u h_{p} \leqslant u h ; u h_{p}, u h \in H$.

$-c a_{1} \leqslant T_{d} ; c a_{1}, T_{d} \in D G$.

$-\left\{m p_{1}\right\} \subseteq\left\{m p_{1}, m p_{2}\right\} ;\left\{m p_{1}\right\},\left\{m p_{1}, m p_{2}\right\} \subseteq M P$.

Example 2. A patient healthcare trajectory can be considered as a set of hospitalisations ordered over time. $\left\langle\left(u h_{p}, c a_{1},\left\{m p_{1}, m p_{2}\right\}\right),\left(g h_{l}, r_{1},\left\{m p_{2}\right\}\right)\right\rangle$ represents a patient trajectory with two hospitalizations. The patient was first admitted in the university hospital $u h_{p}$ for a lung cancer $c a_{1}$, and underwent procedures $m p_{1}$ and $m p_{2}$. Then he visited the general hospital $g h_{l}$ for a pneumonitis $r_{1}$ where he underwent procedure $m p_{2}$. 
Definition 3. (Sequence)

A sequence is represented as $S=\left\langle S_{1}, S_{2}, \ldots, S_{l}\right\rangle$ is a set of elementary sequences $S_{i}$ ordered by the temporal order relation $<_{t}$, such as $S_{1}<_{t} S_{2}<_{t}$ $S_{3} \ldots<_{t} S_{l}$.

Definition 4. (Ordering of sequences)

Given two sequences $S=\left\langle S_{1}, S_{2}, \ldots, S_{l}\right\rangle$ and $T=\left\langle T_{1}, T_{2}, \ldots, T_{l^{\prime}}\right\rangle, S$ is more specific than $T$, denoted by $S \leq_{s} T$, if there exist a set of indices $1 \leq i_{1}<i_{2}<$ $\ldots<i_{l^{\prime}} \leq l$ such that $S_{j} \leq_{e} T_{i_{j}}$ for all $j=1 \ldots l^{\prime}$ and $l^{\prime} \leqslant l$. The most specific sequence is also the longest sequence as $l^{\prime} \leqslant l$.

Example 3. $S=\left\langle\left(u h_{p}, c a_{1},\left\{m p_{1}, m p_{2}\right\}\right),\left(g h_{l}, r_{1},\left\{m p_{2}\right\}\right)\right\rangle$ is a sequence with two elementary sequences $S_{1}=\left(u h_{p}, c a_{1},\left\{m p_{1}, m p_{2}\right\}\right)$ and $S_{2}=\left(g h_{l}, r_{1},\left\{m p_{2}\right\}\right)$ where $S_{1}<_{t} S_{2}$. The sequence $\mathrm{S}$ is more specific than $T=\left\langle\left(u h, T_{d},\left\{m p_{1}\right\}\right)\right\rangle$, $S \leq_{s} T$, as $\left(u h_{p}, c a_{1},\left\{m p_{1}, m p_{2}\right\}\right) \leq_{e}\left(u h, T_{d},\left\{m p_{1}\right\}\right)$.

A set of m sequences $S_{D B}=\left\{S^{1}, S^{2}, \ldots, S^{m}\right\}$ is called hereafter a "sequential database". An example is given in Table 1 where there are four sequences describing four patient trajectories.

Definition 5. (Support of sequence $S$ )

Let $S_{D B}=\left\{S^{1}, \ldots, S^{k}\right\}$ a sequential database. The support of a sequence $S$, denoted by support ${ }_{s}(S)$ is defined as follows:

$$
\operatorname{support}_{s}(S)=\left|\left\{S^{i} \in S_{D B} ; S^{i} \leq_{s} S\right\}\right|
$$

Definition 6. (Sequential pattern)

Given a positive integer $\sigma$ as a minsup threshold and a sequential database $S_{D B}$, the sequence $S$ is called a sequential pattern in $S_{D B}$ iff $\operatorname{support}_{s}(S) \geq \sigma$.

Example 4. The sequence $S=\left\langle\left(u h_{p}, c a,\left\{m p_{1}, m p_{2}\right\}\right),\left(g h_{l}, r,\{\}\right)\right\rangle$ has a support equals to 3 (i.e, $\operatorname{support}_{s}(S)=3$ ) in the sequential database $S_{D B}$ (see Table 1). It is a sequential pattern according to minsup threshold equals to 3 (i.e, $\sigma=3$ ).

\begin{tabular}{|c|c|}
\hline Patients & Trajectories \\
\hline$S^{1}$ & $\left\langle\left(u h_{p}, c a_{1},\left\{m p_{1}, m p_{2}\right\}\right),\left(u h_{p}, c a_{1},\left\{m p_{1}\right\}\right),\left(g h_{l}, r_{1},\left\{m p_{3}\right\}\right)\right\rangle$ \\
\hline$S^{2}$ & $\left\langle\left(u h_{n}, c a_{1},\left\{m p_{4}\right\}\right),\left(u h_{p}, c a_{2},\left\{m p_{1}, m p_{2}\right\}\right),\left(g h_{l}, r_{1},\left\{m p_{2}\right\}\right)\right\rangle$ \\
\hline$S^{3}$ & $\left\langle\left(u h_{n}, c a_{3},\left\{m p_{4}\right\}\right),\left(g h_{l}, r_{2},\left\{m p_{3}\right\}\right)\right\rangle$ \\
\hline$S^{4}$ & $\left\langle\left(u h_{p}, c a_{2},\left\{m p_{1}, m p_{2}\right\}\right),\left(g h_{p}, r_{2},\left\{m p_{3}\right\}\right),\left(g h_{l}, r_{2},\left\{m p_{2}\right\}\right)\right\rangle$ \\
\hline
\end{tabular}

Table 1: An example of a database of patient trajectories. 


\section{Mining Sequential Patterns}

\subsection{Most specific sequential patterns}

In this section, we present the problem of mining the most specific sequential patterns. Given a sequential database, mining all possible frequent patterns results in a huge amount of information that is difficult to manage from the analyst point of view. To overcome this problem, we extract a set of sequential patterns that are not only frequent but also as specific as possible. This second constraint allows to reduce the volume of the final result discarding redundant patterns. An extracted pattern is called a "most specific sequential pattern".

Definition 7. (Most Specific Sequential Pattern (MSSP))

Given a positive integer $\sigma$ as minsup threshold and a sequential database $S_{D B}$, a sequence $S$ is a most specific sequential pattern in $S_{D B}$ or MSSP if and only if $\operatorname{support}_{s}(S) \geq \sigma$ and there does not exist any sequence $T$ such that $T \leq_{S} S$ with support $_{s}(S)=\operatorname{support}_{s}(T) \geq \sigma$

Actually, frequency is monotone (i.e whenever $\mathrm{S}$ is frequent, any generalization of $\mathrm{S}$ is also frequent). For example, if $S=\left\langle\left(u h_{p}, c,\left\{m p_{1}, m p_{2}\right\}\right)\right\rangle$ is frequent then $T=\left\langle\left(u h, c,\left\{m p_{1}\right\}\right)\right\rangle$ which is more general than $S$ is also frequent. Thus, the most specific sequential patterns are sufficient to retrieve all sequential patterns.

Example 5. Let $\sigma=3$ (i.e. a sequence is frequent if it appears at least three times in $\left.S_{D B}\right)$. The sequence $S=\left\langle\left(u h, c a,\left\{m p_{1}\right\}\right)\right\rangle$ is frequent but is not the most specific one because the sequence $T=\left\langle\left(u h_{p}, c a,\left\{m p_{1}, m p_{2}\right\}\right)\right\rangle$ is frequent and verifies $T \leq_{s} S$ and $\operatorname{support}_{s}(S)=\operatorname{support}_{s}(T)$. The sequence $T=\left\langle\left(u h_{p}, c a,\left\{m p_{1}, m p_{2}\right\}\right)\right\rangle$ is a most specific frequent sequence in $S_{D B}$ : (i) $T$ is frequent $\left(\operatorname{support}_{s}(S) \geq \sigma\right.$ ) and (ii) there is no other sequence in $S_{D B}$ which is frequent, more specific than $T$ and has the same support of $T$.

The objective of our approach is to extract a set of frequent sequential patterns that are as specific as possible. In the next section, we present the algorithm $M M I S P$ for finding most specific sequential patterns. The basic idea of MMISP consists in transforming the sequential database into an "adapted form" and then to apply a standard algorithm for sequential mining.

\subsection{The MMISP algorithm}

MMISP is based on three steps:

First step (Extraction of frequent elementary sequences)

The algorithm searches for the frequent and specific elementary sequences. It extracts these frequent elementary sequences w.r.t. partial ordering existing between the elements (posets). 


\section{Second step (Transformation)}

In this step, all frequent elementary sequences extracted in the previous step are mapped to an alternate representation. Then, the sequential database is encoded by using this new representation.

Third step (Mining)

In this step, a standard sequential algorithm is applied on the sequential database produced at the preceding step.

\subsubsection{Extracting all frequent elementary sequences}

Firstly, MMISP considers the elementary sequences in all sequences of $S_{D B}$. Actually if the elementary sequence is not frequent in a sequential database $S_{D B}$ it does not belong to an extracted sequential pattern. For example, If we have two sequences:

\begin{tabular}{|l|l|}
\hline$S^{1}$ & $\left\langle\left\{s_{2}\right\}\left\{s_{1}\right\}\left\{s_{3}\right\}\right\rangle$ \\
\hline$S^{2}$ & $\left\langle\left\{s_{2}\right\}\left\{s_{4}\right\}\left\{s_{3}\right\}\right\rangle$ \\
\hline
\end{tabular}

Given a support threshold $\sigma=2$, pattern $\left\langle\left\{s_{2}\right\}\left\{s_{3}\right\}\right\rangle$ is frequent. In this example, $s_{2}$ and $s_{3}$ are frequent items while $s_{1}$ and $s_{4}$ are not frequent items. In the following, we only consider sequential patterns composed of frequent items.

MMISP extracts all the frequent elementary sequences in a sequential database by taking into account the partial order relation between their elements. The support of an elementary sequence $s$ is defined as follows:

Definition 8. (Support of an elementary sequence e)

Let $S_{D B}=\left\{S^{1}, \ldots, S^{m}\right\}$ a sequential database. The support of an elementary sequence $e=\left(e_{1}, e_{2}, \ldots, e_{n}\right)$, denoted by $\operatorname{support}_{e}(e)$, is defined as follows:

$$
\text { support }_{e}(e)=\mid\left\{S^{i} \in S_{D B} ; S^{i}=\left\langle S_{1}^{i}, \ldots, S_{l}^{i}\right\rangle \text { and } \exists j \in[1, . ., l] ; S_{j}^{i} \leq_{e} e\right\} \mid
$$

Example 6. The support of $\left(g h, r,\left\{m p_{3}\right\}\right)$ is 3 in $S_{D B}$ as:

- $S_{3}^{1}=\left(g h_{l}, r_{1},\left\{m p_{3}\right\}\right) \leq_{e}\left(g h, r,\left\{m p_{3}\right\}\right)$.

$-S_{2}^{3}=\left(g h_{l}, r_{2},\left\{m p_{3}\right\}\right) \leq_{e}\left(g h, r,\left\{m p_{3}\right\}\right)$.

$-S_{2}^{4}=\left(g h_{p}, r_{2},\left\{m p_{3}\right\}\right) \leq_{e}\left(g h, r,\left\{m p_{3}\right\}\right)$.

The frequent elementary sequences are ordered over a poset L, denoted by $\left(L, \leq_{e}\right)$, as follows. Firstly, we generate the most general elementary sequences. In the running example, we consider triples of the form $(h, d g, m p)$ and the most general triple is $\left(T_{h}, T_{d},\{\}\right)$ where $T_{h}$ and $T_{d}$ denote the most general items in the posets $H$ and $D$ respectively, and the empty set \{\} denotes the most general item in the set $M P$. Then, we recursively generate new elementary sequences by starting from the most general one. This generation is done by replacing each element $e_{1}, e_{2}, \ldots, e_{n} \in e$ with all of its direct specializations.

The set of all direct specializations of an element $e_{i}$, denoted by $\operatorname{desc}\left(e_{i}\right)$, is defined as follows: 
Definition 9. (Direct specializations of an element $e_{i}$ )

Let $e_{i}$ be an element in the set $D_{i}$. The direct specializations of $e_{i}$, denoted by $\operatorname{desc}\left(e_{i}\right)$, is defined by:

$\operatorname{desc}\left(e_{i}\right)= \begin{cases}\left\{u \in D_{i} ; u \leq e_{i} \text { and } \nexists w \in D_{i} ; u \leq w \text { and } w \leq e_{i}\right\} & \text { if } D_{i} \text { is a poset. } \\ \left\{e_{i} \cup\{u\} ; u \in D_{i} \backslash e_{i}\right\} & \text { if } D_{i} \text { is a set. }\end{cases}$

The set of all direct specializations of an elementary sequence $e$, denoted by $\operatorname{desc}(e)$, is defined as follows:

Definition 10. (Direct specializations of an elementary sequence e)

Let $e=\left(e_{1}, e_{2}, \ldots, e_{n}\right)$ be an elementary sequence. The direct specializations of an elementary sequence e, denoted by $\operatorname{desc}(s)$, is defined by:

$\operatorname{desc}(e)=\left\{\left(e_{1}^{\prime}, e_{2}^{\prime}, \ldots, e_{n}^{\prime}\right) \mid \exists i \in\{1, \ldots, n\}\left(\left(e_{i}^{\prime} \in \operatorname{desc}\left(e_{i}\right)\right)\right.\right.$ and $\left.\left.(\forall j \neq i)\left(e_{j}^{\prime}=e_{j}\right)\right)\right\}$.

Example 7. Given the most general elementary sequence $\mathrm{s}=\left(T_{h}, T_{d},\{\}\right)$, the direct specializations of $T_{h}$ are $u h$ and $g h$, the direct specializations of $T_{d}$ are $r$ and $c$ and the direct specializations of \{\} are $\left\{m p_{1}\right\},\left\{m p_{2}\right\},\left\{m p_{3}\right\}$ and $\left\{m p_{4}\right\}$. Thus, the direct specializations of $\left(T_{h}, T_{d},\{\}\right)$ are $\left(u h, T_{d},\{\}\right),\left(g h, T_{d},\{\}\right),\left(T_{h}, r,\{\}\right)$, $\left(T_{h}, c a,\{\}\right),\left(T_{h}, T_{d},\left\{m p_{1}\right\}\right),\left(T_{h}, T_{d},\left\{m p_{2}\right\}\right),\left(T_{h}, T_{d},\left\{m p_{3}\right\}\right)$ and $\left(T_{h}, T_{d},\left\{m p_{4}\right\}\right)$.

The frequency of an elementary sequence is anti-monotone w.r.t the specificity of elementary sequence, i.e., whenever an elementary sequence e is not frequent, all the specializations of e are also not frequent. For example, $\left(T_{h}, T_{d}\right.$, $\left.\left\{m p_{4}\right\}\right)$ is not frequent when minsup threshold is equal to 3 , then all the specializations of $\left(T_{h}, T_{d},\left\{m p_{4}\right\}\right)$ such as $\left(g h, T_{d},\left\{m p_{4}\right\}\right)$ or $\left(g h, r,\left\{m p_{1}, m p_{4}\right\}\right) \ldots$ are also not frequent. We use this anti-monotonicity to prune the enumeration space and efficiently build the poset $\left(L, \leq_{e}\right)$.

Specialization is applied recursively for each new frequent elementary generated sequence. Figure 2 shows an example of generation of a poset of $\left(L, \leq_{e}\right)$ which is detailed below:

- Considering the most general elementary sequence $\left(T_{h}, T_{d},\{\}\right)$, our approach generates seven new frequent elementary sequences, which are: $\left(u h, T_{d},\{\}\right)$, $\left(g h, T_{d},\{\}\right),\left(T_{h}, r,\{\}\right),\left(T_{h}, c a,\{\}\right),\left(T_{h}, T_{d},\left\{m p_{1}\right\}\right),\left(T_{h}, T_{d},\left\{m p_{2}\right\}\right)$ and $\left(T_{h}\right.$, $\left.T_{d},\left\{m p_{3}\right\}\right)$.

- Based on $\left(u h, T_{d},\{\}\right), M M I S P$ generates $\left(u h_{p}, T_{d},\{\}\right),(u h, c a,\{\}),\left(u h, T_{d}\right.$, $\left.\left\{m p_{1}\right\}\right)$ and $\left(u h, T_{d},\left\{m p_{2}\right\}\right)$.

- Based on $\left(u h_{p}, T_{d},\{\}\right), M M I S P$ generates $\left(u h_{p}, c a,\{\}\right),\left(u h_{p}, T_{d},\left\{m p_{1}\right\}\right)$ and $\left(u h_{p}, T_{d},\left\{m p_{2}\right\}\right)$.

- Based on $\left(u h_{p}, c a,\{\}\right), M M I S P$ generates $\left(u h_{p}, c a,\left\{m p_{1}\right\}\right)$ and $\left(u h_{p}, c a\right.$, $\left.\left\{m p_{2}\right\}\right)$.

- Based on $\left(u h_{p}, c a,\left\{m p_{1}\right\}\right), M M I S P$ generates $\left(u h_{p}, c a,\left\{m p_{1}, m p_{2}\right\}\right)$.

- By using $\left(u h_{p}, c a,\left\{m p_{1}, m p_{2}\right\}\right)$, no any new frequent elementary sequences can be found, thus the generation stops. 


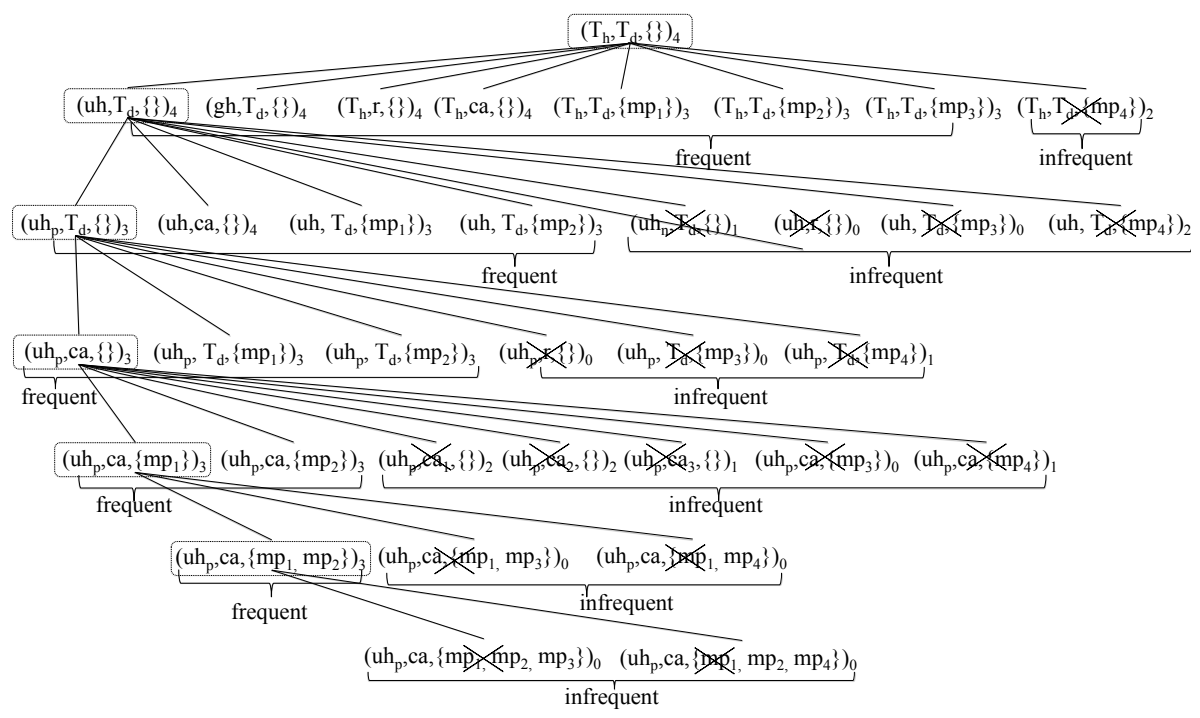

Fig. 2: The steps of generating the elementary sequences in $\left(L, \leq_{e}\right)$ with min$\sup =3$.

Figure 3 shows the poset $\left(L, \leq_{e}\right)$ generated with $\sigma=3$.

As the objective of MMISP is to extract specific sequential patterns, we retain only the most specific elementary sequences in $\left(L, \leq_{e}\right)$. The most specific frequent elementary sequences is defined as follows:

Definition 11. (Most Specific Frequent Elementary Sequence (MSFES))

Given a positive integer $\sigma$ as minsup threshold and a sequential database $S_{D B}$, an elementary sequence $e$ is a most specific frequent elementary sequence in $S_{D B}$ or MSFES if and only if support $_{e}(e) \geq \sigma$ and there does not exist any elementary sequence $e^{\prime}$ such that support $e_{e}\left(e^{\prime}\right) \geq \sigma$ and $e^{\prime} \leq_{e} e$.

Table 2 shows the set of most specific frequent elementary sequences which are extracted from $\left(L, \leq_{e}\right)$. Algorithm 1 and 2 describe the two steps for extracting all the frequent elementary sequences and the most specific ones.

\begin{tabular}{|c|c|}
\hline id & $M S F E S$ \\
\hline 1 & $\left(u h_{p}, c a,\left\{m p_{1}, m p_{2}\right\}\right)$ \\
\hline 2 & $\left(g h_{l}, r,\{\}\right)$ \\
\hline 3 & $\left(g h, r,\left\{m p_{3}\right\}\right)$ \\
\hline
\end{tabular}

Table 2: The most specific frequent elementary sequences extracted from $\left(L, \leq_{e}\right)$. 

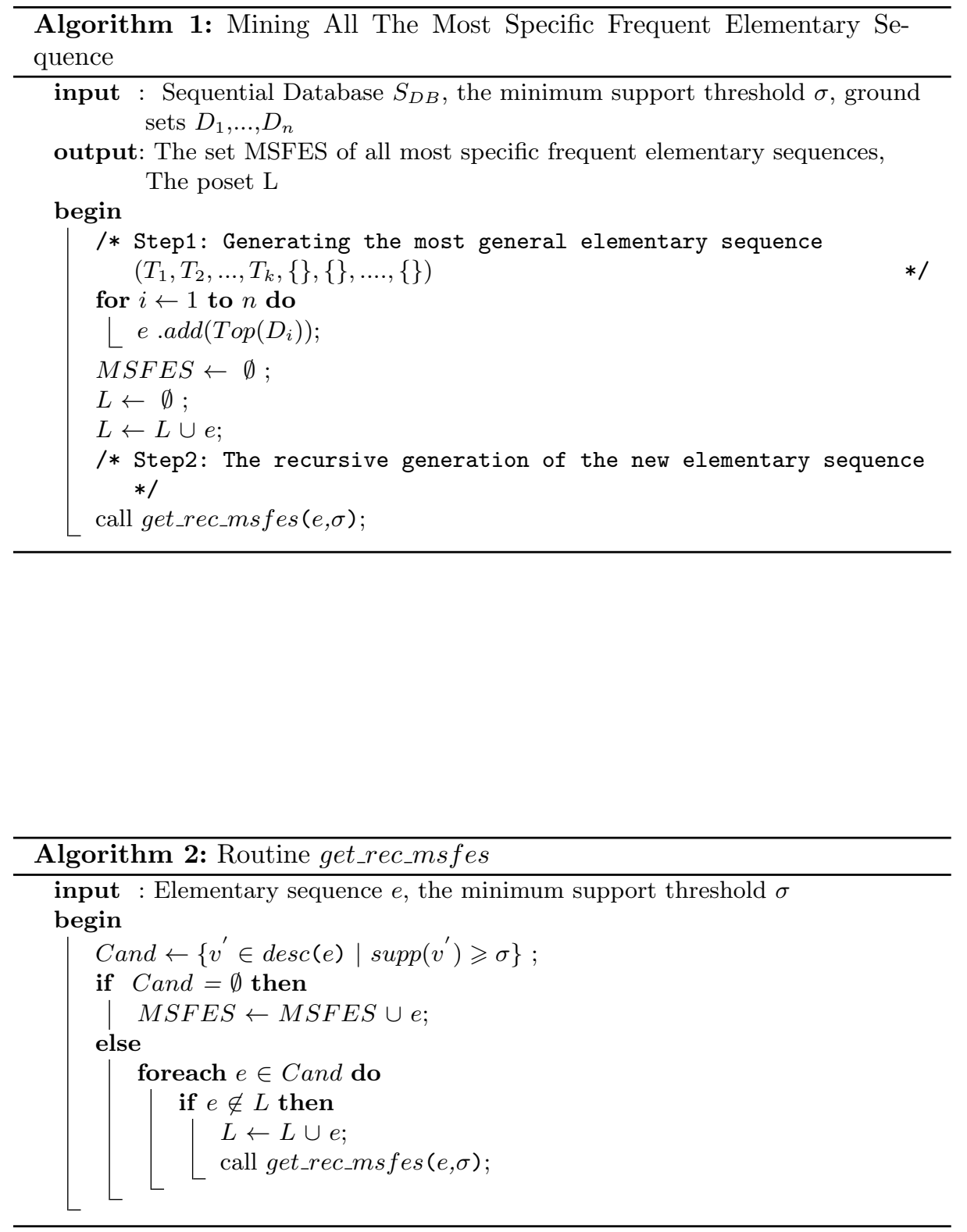


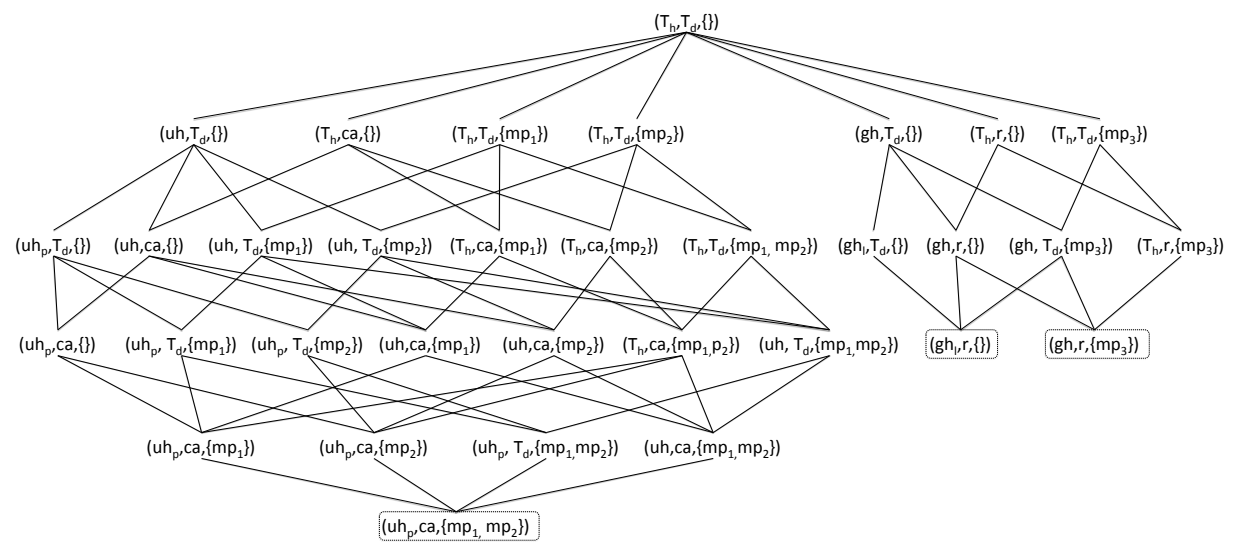

Fig. 3: The poset $\left(L, \leq_{e}\right)$ is generated by taking into account: (i) the sequential database $S_{D B}$ in Table 1 and (ii) the two posets $\mathrm{H}$ and DG in Figure 1 and the set $M P=\left\{m p_{1}, m p_{2}, m p_{3}, m p_{4}\right\}$ with minsup threshold equals to 3 .

\subsubsection{Transformation and mining sequences}

We now study the temporal relation between the extracted specific frequent elementary sequences as follow. Firstly, we replace each elementary sequence in each sequence of $S_{D B}$ with all its generalizations from MSFES set. Given a sequence $S=\left\langle S_{1}, \ldots, S_{n}\right\rangle$ in $S_{D B}$ the replacement consists in substituting each elementary sequence $S_{i}$ in $S$ by several elementary sequences $e \in M S F E S$ such that $S_{i} \leqslant e$.

Example 8. The sequence $S^{1}$ in $S_{D B},\left\langle\left(u h_{p}, c a_{1},\left\{m p_{1}, m p_{2}\right\}\right),\left(u h_{p}, c a_{1},\left\{m p_{1}\right\}\right)\right.$, $\left.\left(g h_{l}, r_{1},\left\{m p_{3}\right\}\right)\right\rangle$ is transformed into $\left\langle\left\{\left(u h_{p}, c a,\left\{m p_{1}, m p_{2}\right\}\right)\right\},\left\{\left(g h_{l}, r,\{\}\right),(g h\right.\right.$, $\left.\left.\left.r,\left\{m p_{3}\right\}\right)\right\}\right\rangle$ :

- $S_{1}^{1}=\left(u h_{p}, c a_{2},\left\{m p_{1}, m p_{2}\right\}\right)$ is replaced by $\left(u h_{p}, c a,\left\{m p_{1}, m p_{2}\right\}\right)$ from MSFES set in Table 2, with $\left(u h_{p}, c a_{2},\left\{m p_{1}, m p_{2}\right\}\right) \leqslant_{e}\left(u h_{p}, c a,\left\{m p_{1}, m p_{2}\right\}\right)$.

- $S_{2}^{1}=\left(u h_{p}, c a_{1},\left\{m p_{1}\right\}\right)$, none of the elementary sequences in MSFES set are more general than $S_{2}^{1}$.

- $S_{3}^{1}=\left(g h_{l}, r_{1},\left\{m p_{3}\right\}\right)$ is replaced by $\left(g h_{l}, r,\{\}\right)$ and $\left(g h, r,\left\{m p_{3}\right\}\right)$ from $M S F E S$ set.

Table 3 shows the transformation of $S_{D B}$ in Table 1 based on the set of all most specific frequent elementary sequences MSFES in Table 2.

In a classical sequential pattern mining algorithm, the sequential database to be mined should be a set of pairs $($ sid, $s$ ) where sid is a unique sequence identifier and $s$ is a sequence of itemsets. Thus $S_{D B}$ in Table 3 is transformed as follows:

- Each elementary sequence in the MSFES is assigned a unique id which is used during the mining (see Table 2) . 


\begin{tabular}{|c|c|}
\hline Patients & Trajectories \\
\hline$S^{1}$ & $\left\langle\left\{\left(u h_{p}, c a,\left\{m p_{1}, m p_{2}\right\}\right)\right\},\left\{\left(g h_{l}, r,\{\}\right),\left(g h, r,\left\{m p_{3}\right\}\right)\right\}\right\rangle$ \\
\hline$S^{2}$ & $\left\langle\left\{\left(u h_{p}, c a,\left\{m p_{1}, m p_{2}\right\}\right)\right\},\left\{\left(g h_{l}, r,\{\}\right)\right\}\right\rangle$ \\
\hline$S^{3}$ & $\left\langle\left\{\left(g h_{l}, r,\{\}\right),\left(g h, r,\left\{m p_{3}\right\}\right)\right\}\right\rangle$ \\
\hline$S^{4}$ & $\left\langle\left\{\left(u h_{p}, c a,\left\{m p_{1}, m p_{2}\right\}\right)\right\},\left\{\left(g h, r,\left\{m p_{3}\right\}\right)\right\},\left\{\left(g h_{l}, r,\{\}\right)\right\}\right\rangle$ \\
\hline
\end{tabular}

Table 3: Transforming the patient trajectories in Table 1 by using the set of all most specific frequent elementary sequences in Table 2.

- For each sequence $S^{i}$ in the transformed database (see Table 3) and for each elementary sequence $T$ in $S_{j}^{i} ; S_{j}^{i} \in S^{i}, \mathrm{~T}$ is replaced by its id.

Example 9. The sequence $S^{1}=\left\langle\left\{\left(u h_{p}, c a,\left\{m p_{1}, m p_{2}\right\}\right)\right\},\left\{\left(g h_{l}, r,\{\}\right),(g h, r\right.\right.$, $\left.\left.\left.\left\{m p_{3}\right\}\right)\right\}\right\rangle$ in Table 3 is transformed into $\langle\{1\},\{2,3\}\rangle$ such as:

- $\left(u h_{p}, c a,\left\{m p_{1}, m p_{2}\right\}\right)$ in $S^{1}$ has id 1 in Table 2.

- $\left(g h_{l}, r,\{\}\right)$ in $S^{1}$ has id 2 in Table 2.

- $\left(g h, r,\left\{m p_{3}\right\}\right)$ in $S^{1}$ has id 3 in Table 2.

Table 4 shows the transformation sequential database of Table 3 by using the identifiers of all most specific frequent elementary sequences MSFES in Table 2.

\begin{tabular}{|c|c|}
\hline Patients & Trajectories \\
\hline$S^{1}$ & $\langle\{1\}\{2,3\}\rangle$ \\
\hline$S^{2}$ & $\langle\{1\}\{2\}\rangle$ \\
\hline$S^{3}$ & $\langle\{2,3\}\rangle$ \\
\hline$S^{4}$ & $\langle\{1\}\{3\}\{2\}\rangle$ \\
\hline
\end{tabular}

Table 4: Transformed database in Table 3

Then in MMISP, we use CloSpan [12] as the sequential pattern mining algorithm. Table 5 displays all sequential patterns in their transformed format and the frequent patient trajectories in which identifiers are replaced with their actual values, with $\sigma=3$.

\section{Experiments}

We conduct experiments on both real and synthetic datasets. The MMISP algorithm is implemented in Java and the experiments are carried out on a MacBook Pro with a $2.5 \mathrm{GHz}$ Intel Core i5, 4GB of RAM Memory running OS X 10.6.8. Extraction of sequential patterns is based on the public implementation of CloSpan algorithm [12] supplied by the IlliMine ${ }^{4}$ toolkit.

\footnotetext{
${ }^{4}$ http://illimine.cs.uiuc.edu/
} 


\begin{tabular}{|c|c|c|}
\hline Frequent sequential patterns & Frequent patient trajectory patterns & Support \\
\hline$\langle\{2\}\rangle$ & $\left\langle\left(g h_{l}, r,\{\}\right)\right\rangle$ & 4 \\
\hline$\langle\{3\}\rangle$ & $\left\langle\left(g h, r,\left\{m p_{3}\right\}\right)\right\rangle$ & 3 \\
\hline$\langle\{1\}\{2\}\rangle$ & $\left\langle\left(u h_{p}, c a,\left\{m p_{1}, m p_{2}\right\}\right),\left(g h_{l}, r,\{\}\right)\right\rangle$ & 3 \\
\hline
\end{tabular}

Table 5: All the most specific sequential patterns extracted from $S_{D B}$ in Table 1 with $\sigma=3$.

\subsection{Healthcare Trajectory}

\subsubsection{Mining healthcare trajectories}

In order to assess the effectiveness of our approach, we run several experiments on the PMSI system for describing and analyzing patient trajectories. In PMSI, each hospitalization is characterized by the following dimensions: hospital, principal diagnosis and procedures delivered during the stay.

The hospital dimension is associated with a geographical poset of 4 levels: root (France), administrative region, administrative departement and hospital. As illustrated in Figure 4, University Hospital of Nancy (coded as 540002078) is a hospital in Meurthe-et-Moselle and Meurthe-et-Moselle is a department in Lorraine which is a region of France. The number of nodes in this taxonomy is 151 nodes.

Principal Diagnosis could be described at 5 levels of the $10^{\text {th }}$ International classification of Diseases (ICD10): root, chapter, block, 3 characters and 4 characters. As illustrated in Figure 5, chapters such as Neoplasms have specializations: block $C 30-C 39$ which is a malignant neoplasms of respiratory, block $C 50-C 50$ which is a malignant neoplasms of breast etc. The block $C 30-C 39$ has specializations: malignant neoplasm of larynx (coded as C34), malignant neoplasm of bronchus and lung (coded as C32), etc. C34 (Lung cancer) has specializations: $C 340$ is a cancer of the main bronchus, $C 341$ is a cancer of upper lobe etc. The number of nodes in the disease taxonomy is 1543 nodes.

Procedures were represented by their first $\mathrm{CCAM}^{5}$ code. For example, $Z B Q K$ is a chest radiography, GFFA is a pneumonectomy etc.

Our dataset contains 828 patients suffering from lung cancer and living in Lorraine Region, in the East of France. Table 6 shows an example of care trajectories for 3 patients. For example, Patient $_{1}$ has two hospitalizations. He was admitted in the University Hospital of Nancy (coded as 540002078), for a Lung cancer (coded as C341), and underwent a chest radiography (coded as $Z B Q K$ ). Then, he was hospitalized in a private clinic in Metz (coded as 570023630), for a chemotherapy session (coded as 251 ) where he had a chest radiography and pneumonectomy (coded as GFFA).

5 "Classification Commune des Actes Médicaux": the French classification of medical and surgical procedures. 


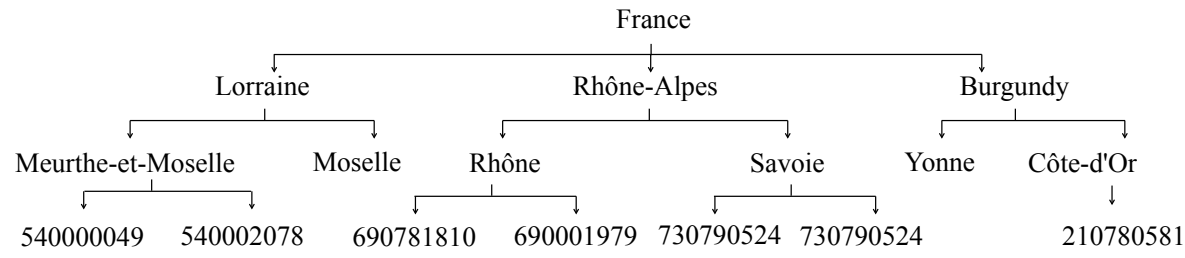

Fig. 4: A geographical poset of the healthcare institution

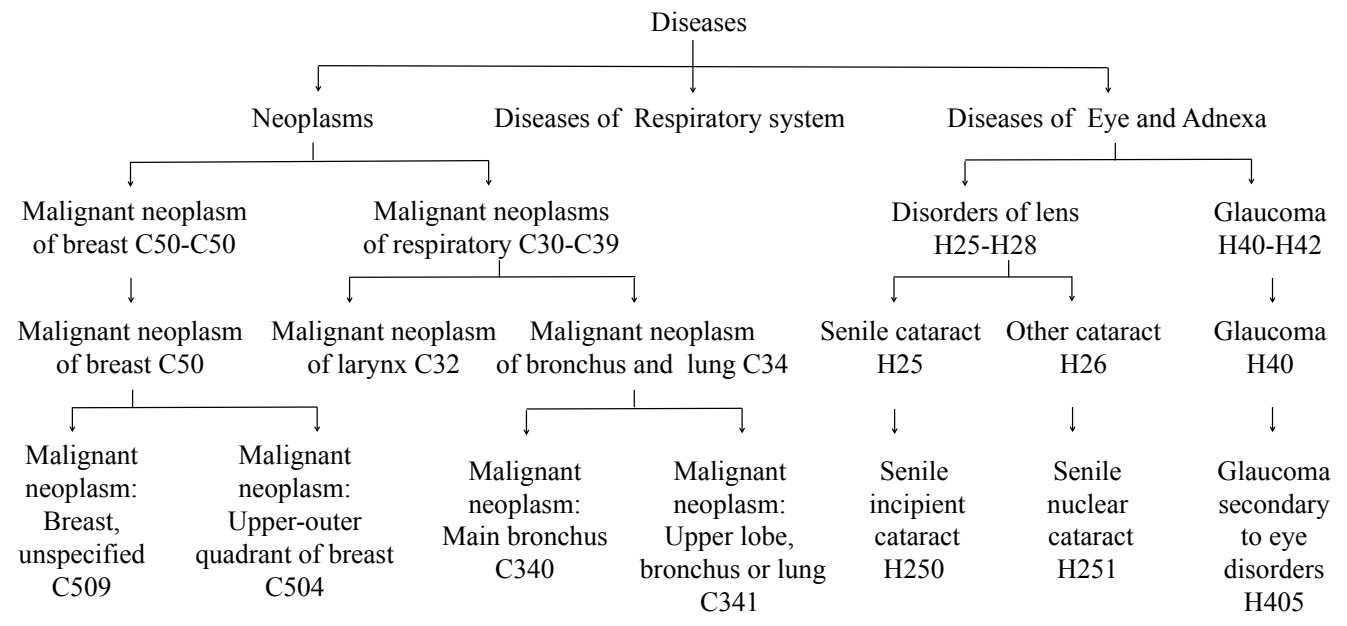

Fig. 5: A disease poset 
As a characterization of the care trajectory database, Figure 6 shows the distribution of the length of healthcare trajectories in our dataset, the median length is of 11 stays.

\begin{tabular}{|l|c|}
\hline Patients & Trajectories \\
\hline \hline Patient $^{1}$ & $\langle(C 341,540002078,\{Z B Q K\}),(Z 51,570023630,\{Z B Q K, G F F A\}), \ldots\rangle$ \\
\hline Patient $^{2}$ & $\langle(C 770,100000017,\{Z B Q K\}),(C 770,210780581,\{Z Z Q K, Y Y Y Y\}), \ldots\rangle$ \\
\hline Patient $^{3}$ & $\langle(H 259,210780110,\{Y Y Y Y\}),(H 259,210780110,\{Z Z Q K\}), \ldots\rangle$ \\
\hline
\end{tabular}

Table 6: Care trajectories of 3 patients

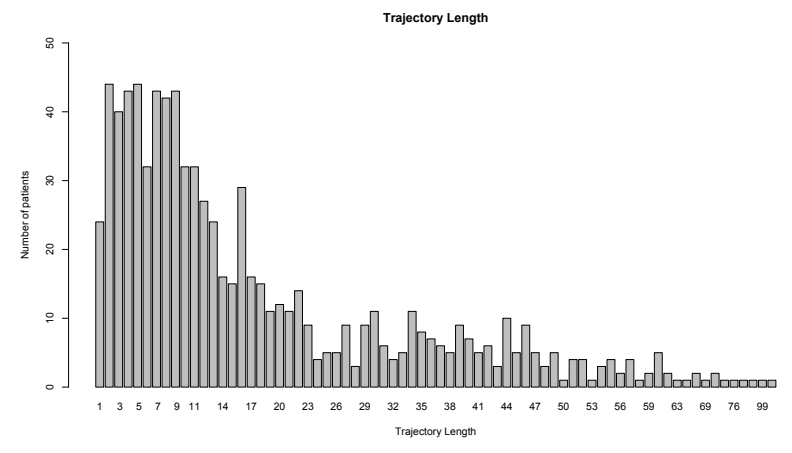

Fig. 6: Distribution length of the patient trajectories

The support value is set to 40 patients (i.e. $\sigma=5 \%$ ) for this experiment . MMISP generates 615 different frequent trajectories. Table 7 shows some extracted patterns. Pattern \#1 can be interpreted as follows: $5 \%$ of patients had a hospitalization in Meurthe et Moselle department for any kind of lung cancer (coded as C34). They underwent three medical procedures: chest radiography (coded as ZBQK) with an electrocardiography (coded as DEQP) and a therapeutic procedure on blood (coded as FELF). Pattern \#2 shows that $8 \%$ of patients had a hospitalization in Lorraine, because of poisoning.

This kind of information helps healthcare managers and decision makers in planning and organizing healthcare resources at regional level. Besides, sequential patterns can be seen as condensed representations of the care trajectories.

\subsubsection{MMISP versus Standard sequential pattern mining method}

In this section, we compare MMISP with a standard sequential pattern mining method such as CloSpan. All standard sequential pattern mining algorithms 


\begin{tabular}{|c|c|c|}
\hline Pattern & Trajectories & support \\
\hline \hline Pattern $^{1}$ & $\langle($ Meurthe et Moselle, C34, $\{Z$ ZQK $, F E L F, D E Q P\})\rangle$ & $5 \%$ \\
\hline Pattern $^{2}$ & $\langle($ Lorraine, Poisoning $)\rangle$ & $8 \%$ \\
\hline Pattern $^{3}$ & $\langle(540003019, Z 510,\{Z Z N L\}),(540003019, Z 510,\{Z Z N L\})\rangle$ & $14 \%$ \\
\hline
\end{tabular}

Table 7: 3-Care trajectories extracted by MMSIP

requires the mined dataset to be mined is composed of pairs of the form (id,seq), where $i d$ is a sequence identifier and $s e q$ is a sequence of itemsets. So, we replace each sequence $S^{i}$ in $S_{D B}$ with an extended-sequence $S^{\prime}{ }^{i}$. Each elementary sequence of a sequence $S^{i}$ is transformed into a single itemset by replacing each element in the elementary sequence with all its ancestors. For example, with the posets shown in Figure 1, an elementary sequence $\left(u h_{p}, c a_{1},\left\{m p_{1}, m p_{2}\right\}\right)$ would be replaced with $\left\{T_{h}, u h, u h_{p}, T_{d}, c a, c a_{1}, m p_{1}, m p_{2}\right\}$. Table 8 shows the extended-sequential database $S_{D B}^{\prime}$ of the sequential database $S_{D B}$ in Table 1 . Then, we apply CloSpan as a sequential pattern mining algorithm on the extended-sequential database. This way of managing taxonomies has been used in GSP which is proposed by Srikant and Agrawal [11].

\begin{tabular}{|c|c|}
\hline Patients & Extended sequence \\
\hline$S^{1}$ & $\left\langle\left\{T_{h}, u h, u h_{p}, T_{d}, c a, c a_{1}, m p_{1}, m p_{2}\right\},\left\{T_{h}, u h, u h_{p}, T_{d}, c a, c a_{1}, m p_{1}\right\},\left\{T_{h}, g h, g h_{l}, T_{d}, r, r_{1}, m p_{3}\right\}\right\rangle$ \\
\hline$S^{2}$ & $\left\langle\left\{T_{h}, u h, u h_{n}, T_{d}, c a, c a_{1}, m p_{4}\right\},\left\{T_{h}, u h, u h_{p}, T_{d}, c a, c a_{2}, m p_{1}, m p_{2}\right\},\left\{T_{d}, g h, g h_{l}, T_{d}, r, r_{1}, m p_{2}\right\}\right\rangle$ \\
\hline$S^{3}$ & $\left\langle\left\{T_{h}, u h, u h_{n}, T_{d}, c a, c a_{3}, m p_{4}\right\},\left\{T_{h}, g h, g h_{l}, T_{d}, r, r_{2}, m p_{3}\right\}\right\rangle$ \\
\hline$S^{4}$ & $\left\langle\left\{T_{h}, u h, u h_{p}, T_{d}, c a, c a_{2}, m p_{1}, m p_{2}\right\},\left\{T_{h}, g h, g h_{p}, T_{d}, r, r_{2}, m p_{3}\right\},\left\{T_{h}, g h, g h_{l}, T_{d}, r, r_{2}, m p_{2}\right\}\right\rangle$ \\
\hline
\end{tabular}

Table 8: An extended sequential database of patient trajectories in Table 1.

Our main goal is to evaluate the quality of the patterns mined with MMISP and its performance compared to the "naive" approach using CloSpan. So, we transform the 828 patients trajectories, then we apply the two approaches using several minimum support threshold ranging from $100 \%$ to $5 \%$. Figure 7 reports the running time and the number of pattens according to different values of support threshold for both CloSpan and MMISP.

Actually, CloSpan cannot finish its calculations for support threshold less than $50 \%$ because the transformation increases number of items in itemsets and generates a large number of similar sequences. By contrast, MMISP runs in acceptable time for support as low as $5 \%$.

MMISP is able to extract condensed patterns w.r.t. the ones mined by CloSpan. For example, the sequential pattern $\left\langle\left\{T_{h}, u h, u h_{p}, T_{d}, c a, m p_{1}, m p_{2}\right\}\right.$ $\left.\left\{T_{h}, g h, g h_{l}, T_{d}, r\right\}\right\rangle$ generated by CloSpan contains redundant information as a hospitalization containing $u h_{p}$ will also contain $T_{h}$ and $u h$. MMISP is not 


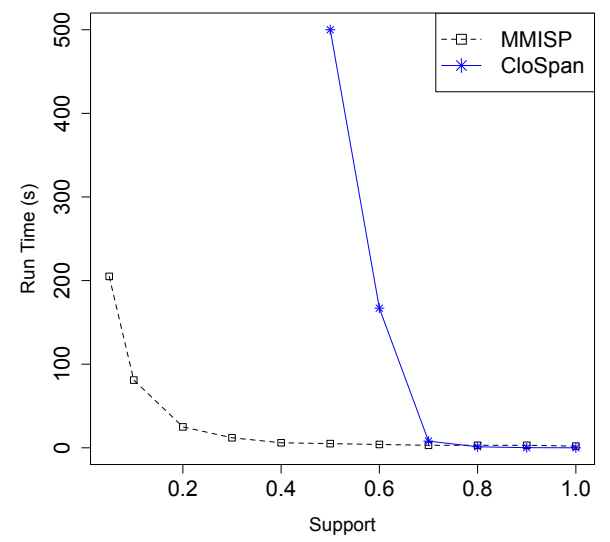

(a) Runtime sequences over frequency threshold

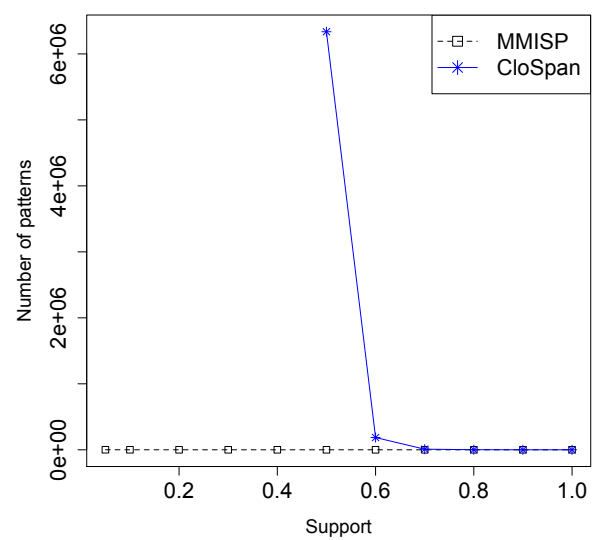

(b) Number patterns over frequency threshold

Fig. 7: $M M I S P$ versus CloSpan

concerned by this pattern because it extracts just the most specific frequent elementary sequence in the first step of the algorithm.

CloSpan extracts all the sequential patterns while MMISP generates just the more specific ones. For example, if $\left\langle\left(u h_{p}, c a,\left\{m p_{1}, m p_{2}\right\}\right)\right\rangle$ is a sequential pattern. MMISP does not extract the patterns which are more general such as $\left\langle\left(u h, T_{d},\left\{m p_{1}\right\}\right)\right\rangle$ while CloSpan extracts both the general and specific ones. Figure 7 shows the differences between the number of sequential patterns extracted by CloSpan and MMISP. For example, with a support threshold of $50 \%, M M I S P$ extracts 150 sequential patterns while CloSpan extracts 6335683 sequential patterns.

Finally, we may conclude that:

- MMISP is more efficient than CloSpan over extended-sequential database with low support threshold.

- The sequential patterns extracted by CloSpan require post processing while this is not the case with MMISP.

- MMISP extracts just the most specific sequential patterns while CloSpan extracts both general and specific ones. This means that CloSpan extracts a huge number of sequential patterns. Analyzing all these sequential patterns is not an easy task for healthcare managers and decision makers.

\subsection{3 $M M I S P$ versus $M^{3} S P$}


Another experiment was carried out for comparing $M^{3} S P$ with $M M I S P$. Our main goal is to evaluate the effectiveness of sequential patterns mined by $M M I S P$ compared to the ones extracted by $M^{3} S P$. For this purpose, we applied $M^{3} S P$ with hospital, diagnosis and medical procedures as analysis dimensions. The support value is set to 40 patients (i.e. $\sigma=5 \%$ ). Table 9 reports an example of the extracted patterns with $M^{3} S P$ and $M M I S P$.

Firstly, we observe that $M M I S P$ is able to extract condensed patterns w.r.t. the ones mined by $M^{3} S P$. For example, 48 sequential patterns, Pattern \#1,..., Pattern \#48, generated by $M^{3} S P$ are summarized by 3 sequential patterns, Pattern \#50, Pattern \#51 and Pattern \#52, extracted by MMISP (see Table 9). This shows that the rigid structure of multidimensional item assumed by $M^{3} S P$ limits the expressivity of the results.

Besides that, in $M^{3} S P$, several dimensions can be repeated in the same hospitalization. For example, in $M^{3} S P$, Pattern \#48 represents one hospitalization including 9 multidimensional items. Each multidimensional item is associated with the same value of hospital and diagnosis (570000588 and C341) and different values of medical procedures. By contrast, Pattern \#50 extracted by MMISP represents the same trajectory as Pattern \#48. Pattern \#50 has one elementary sequence with three elements: hospital 570000588, diagnosis $C 341$ and a set of medical procedure $\{Z B Q K, D E Q P, G F F A, G L L D, G E L D$, $Z Z Q K, G E L E, F C F A, A G L B\}$. Pattern \#50 is much more compact and informative than Pattern \#48.

Given a minsup threshold, MMISP extracts sequential patterns that are not found by $M^{3} S P$. For instance, Pattern \#53 extracted by MMISP is not found by $M^{3} S P$. This is due to the fact that MMISP extracts new frequent elementary sequences not extracted by $M^{3} S P$. For instance $e_{1}=($ Lorraine, Diseases, $\{G E Q E, A C Q H, Z C Q H\})$ and $e_{2}=($ Lorraine,Diseases of the respiratory, $\{A C Q H\})$ are extracted by MMISP. As $e_{1}$ and $e_{2}$ are frequent and not comparable (i.e. $e_{1} \Varangle_{e} e_{2}$ and $\left.e_{2} \Varangle_{e} e_{1}\right), M^{3} S P$ extracts only (Lorraine, Diseases of the respiratory, ACQH) and not (Lorraine, Diseases, ACQH) as (Lorraine, Diseases of the respiratory, $A C Q H$ ) is more specific than (Lorraine, Diseases, $A C Q H)$.

From a quantitative point of view, MMISP extracts 803 frequent elementary sequences with 615 sequential patterns while $M^{3} S P$ extracts 331 multidimensional items with 470 multidimensional sequential patterns. The execution time of $M^{3} S P$ is about 82 seconds while MMISP takes about 98 seconds.

Finally, we may conclude that:

- Several sequential patterns generated by $M^{3} S P$ can be summarized by only one sequential pattern mined by MMISP.

- Several multidimensional items generated by $M^{3} S P$ can be summarized by only one elementary sequence in MMISP.

- One elementary sequence in $M M I S P$ represents one hospitalization in the trajectory while one multidimensional item in $M^{3} S P$ represents only a part of hospitalization in the trajectory. 
- Some sequential patterns can be extracted by MMISP while they cannot be extracted by $M^{3} S P$.

\begin{tabular}{|c|c|c|}
\hline Methods & & Trajectory Patterns \\
\hline \multirow{4}{*}{$M^{3} S P$} & 1 & $\langle\{(570000588, C 341, G F F A)(570000588, C 341, Z Z Q K)\}\rangle$ \\
\hline & 2 & $\langle\{(570000588, C 341, D E Q P)(570000588, C 341, G F F A)(570000588, C 341, Z Z Q K)\}\rangle$ \\
\hline & 48 & $\begin{array}{c}\langle\{(570000588, C 341, Z B Q K)(570000588, C 341, D E Q P)(570000588, C 341, G F F A) \\
(570000588, C 341, G L L D)(570000588, C 341, G E L D)(570000588, C 341, Z Z Q K) \\
(570000588, C 341, G E L E)(570000588, C 341, F C F A)(570000588, C 341,, A G L B)\}\rangle \\
\end{array}$ \\
\hline & 49 & $\langle($ Lorraine, Diseases of the respiratory, $A C Q H)\rangle$ \\
\hline \multirow[t]{2}{*}{ MMISP } & \begin{tabular}{|l|l|}
50 \\
51 \\
52 \\
\end{tabular} & $\begin{array}{c}\langle\{(570000588, C 341,\{Z B Q K, D E Q P, G F F A, G L L D, G E L D, Z Z Q K, G E L E, F C F A, A G L B\}\rangle \\
\langle(570000588, C 341,\{D E Q P, G E L D, G E L E, Z Z Q K, A G L B, G L L D, G F F A\})\rangle \\
\langle(570000588, C 341,\{Z B Q K, D E Q P, G E L D, G E L E, Z Z Q K, G L L D, G F F A\})\rangle \\
\end{array}$ \\
\hline & 53 & $\langle($ Lorraine, Diseases,$\{G E Q E, A C Q H, Z C Q H\})\rangle$ \\
\hline
\end{tabular}

Table 9: Some patterns obtained by $M^{3} S P$ and MMISP.

\subsubsection{MMISP ${ }^{+}$}

In our approach, the fundamental step is the first one which is "extraction of frequent elementary sequences", because it provides all elements that will occur in sequences to be mined. In this step, MMISP extracts only the most specific frequent elementary sequences from the poset $\left(\mathrm{L}, \leq_{e}\right)$. As a result, it is impossible to find a sequential pattern that contains an elementary sequence which is comparable with another elementary sequence in the same pattern or in the another one. Formally, the sequential patterns extracted by MMISP have the following property: for any two sequential patterns $S P^{1}=\left\langle S P_{1}^{1}, S P_{2}^{1}, \ldots, S P_{k^{1}}^{1}\right\rangle$ and $S P^{2}=\left\langle S P_{1}^{2}, S P_{2}^{2}, \ldots, S P_{k^{2}}^{2}\right\rangle$, we have :

$$
\nexists(i, j) ; S P_{i}^{1}<_{e} S P_{j}^{2}
$$

For example, in Table 1 the pattern $\left\langle(u h, c a,\{\}),\left(g h_{l}, r,\{\}\right)\right\rangle$ is frequent according to minsup threshold equals 3 . This pattern does not appear in the results of MMISP because in the first step MMISP extracts $\left(u h_{p}, c a,\left\{m p_{1}, m p_{2}\right\}\right)$ which is more specific than $(u h, c a,\{\})$ and still frequent. As a result, MMISP does not extract any pattern which includes elementary sequence more general than $\left(u h_{p}, c a,\left\{m p_{1}, m p_{2}\right\}\right)$.

To solve this problem, we propose an extension to our approach which is called $M M I S P^{+}$. In $M M I S P^{+}$, instead of choosing just the most specific elementary sequence in $\left(L, \leq_{e}\right)$, we choose all the elementary sequences in $\left(L, \leq_{e}\right)$. Thus, we replace each elementary sequence in each sequence of $S_{D B}$ with all of its generalizations from $\left(L, \leq_{e}\right)$ and not just with the most specific ones. For example, the elementary sequence $\left(g h_{p}, r_{2},\left\{m p_{3}\right\}\right)$ in the sequence $S^{4}$ in Table 1 is replaced by $\left(g h, r,\left\{m p_{3}\right\}\right),(g h, r,\{\}),\left(g h, T_{d},\left\{m p_{3}\right\}\right),\left(T_{h}, r,\left\{m p_{3}\right\}\right),\left(g h, T_{d},\{\}\right)$, 
$\left(T_{h}, r,\{\}\right),\left(T_{h}, T_{d},\left\{m p_{3}\right\}\right)$ and $\left(T_{h}, T_{d},\{\}\right)$ from the $\left(L, \leq_{e}\right)$ set in Figure 3. Then, we use CloSpan as a sequential pattern mining algorithm on the transformed sequential database.

\begin{tabular}{|c|c|}
\hline Frequent patient trajectory patterns & Support \\
\hline$\left\langle(u h, c a,\{\}),\left(g h_{l}, r,\{\}\right)\right\rangle$ & 4 \\
\hline$\left\langle(u h, c a,\{\}),\left(T_{h}, T_{d},\{\}\right),\left(g h_{l}, r,\{\}\right)\right\rangle$ & 3 \\
\hline$\left\langle(u h, c a,\{\}),\left(g h, r,\left\{m p_{3}\right\}\right)\right\rangle$ & 3 \\
\hline$\left\langle\left(u h_{p}, c a,\left\{m p_{1}, m p_{2}\right\}\right),\left(g h_{l}, r,\{\}\right)\right\rangle$ & 3 \\
\hline
\end{tabular}

Table 10: The sequential patterns extracted from $S_{D B}$ with support threshold equals 3 .

Applying $M M I S P^{+}$generates non condensed patterns w.r.t MMISP. For example, the pattern $\left\langle\left\{\left(T_{h}, T_{d},\{\}\right),\left(u h, T_{d},\{\}\right),\left(T_{h}, c a,\{\}\right),(u h, c a,\{\})\right\}\left\{\left(T_{h}, T_{d}\right.\right.\right.$, \{\}$\left.\left.),\left(g h, T_{d},\{\}\right),\left(T_{h}, r,\{\}\right),\left(g h_{l}, T_{d},\{\}\right),(g h, r,\{\}),\left(g h_{l}, r,\{\}\right)\right\}\right\rangle$ generated by MMISP ${ }^{+}$contains redundant information. as a patient having the hospitalization $(u h, c a,\{\})$ will also have $\left(T_{h}, T_{d},\{\}\right),\left(u h, T_{d},\{\}\right)$ and $\left(T_{h}, c a,\{\}\right)$. Redundancy can be removed by post-processing. Table 10 shows all the sequential patterns extracted from $S_{D B}$ in Table 1 by applying $M M I S P^{+}$. These patterns have been post-processed to remove redundant information.

Figure 8 reports the number of patterns extracted from our dataset (i.e, 828 patient trajectories) according to different values of minsup threshold for applying the two solutions: $M M I S P$ and $M M I S P^{+}$. Actually, $M M I S P^{+}$cannot finish its calculations for support threshold less than $50 \%$. This happens because the CloSpan algorithm (i.e, the third step in $M M I S P^{+}$) cannot process with support threshold less than $50 \%$.

\subsection{Experiments on Synthetic Datasets}

In the second experiment, we study the scalability of the MMISP approach. We consider the number of extracted patterns and the running time with respect several parameters:

- number of dimensions with an associated subsumption relation.

- number of dimensions without any associated subsumption relation.

- number of elementary sequences in each sequence (i.e. sequence length).

- depth of the poset of elements with an associated subsumption relation.

- number of sequences in a sequential database.

In the following, we use the term "set-element" for an element lying in a set and the term "poset-element" for an element lying in a partially ordered set.

The first batch of synthetic data generated contains 1000 sequences defined over 2, 3, 4 and 5 poset-elements and 2 set-elements. Each sequence contains 15 


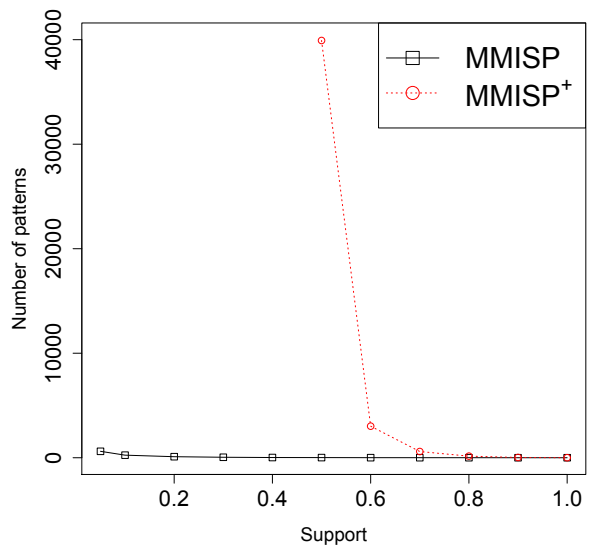

Fig. 8: Number of sequential pattern extracted according to different values of support threshold for both MMISP and MMISP ${ }^{+}$

elementary sequences. Each poset is defined over 3 levels of granularity between its elements. Figure 9 reports the results according to different values of support threshold for different numbers of poset-elements in the elementary sequence. The running time increases for each newly added dimension.

The second batch of generated synthetic data contains 1000 sequences with varying number of set-elements (2, 3, 4 and 5 elements). The sequences have three poset-elements with 3-level of granularity. Figure 10 reports the results according to different values of support threshold for different number of set-elements in the elementary sequence.

In Figure 11, we study the performance of MMISP by considering several levels of granularity within the posets. We generated 1000 sequences defined over 3 poset-elements and 3 set-elements. Each poset is defined over 3, 4, 5, 6 levels of granularity. Each sequence contains 15 elementary sequences. The number of extracted sequential patterns does not change with each newly added level as MMISP extracts only the most specific sequential patterns.

We study the performance of MMISP and the number of extracted sequential patterns with respect the number of sequences in a sequential database and the length of each sequence. Figure 12 shows the running time and the number of patterns extracted for 1000 sequences with 3 poset-elements and 3 set-elements with varying sequence length. Figure 13 shows the running time and the number of sequential patterns extracted for several number of sequences $(1000,2000$, 3000, 4000 and 5000 sequences) also with 3 poset-elements and 3 set-elements. In this experiments, the sequence length is roughly equal to 15 .

Figures 9 - 13 highlight the fact that MMISP is efficient in terms of runtime for a large panel of sequences when varying different parameters. 

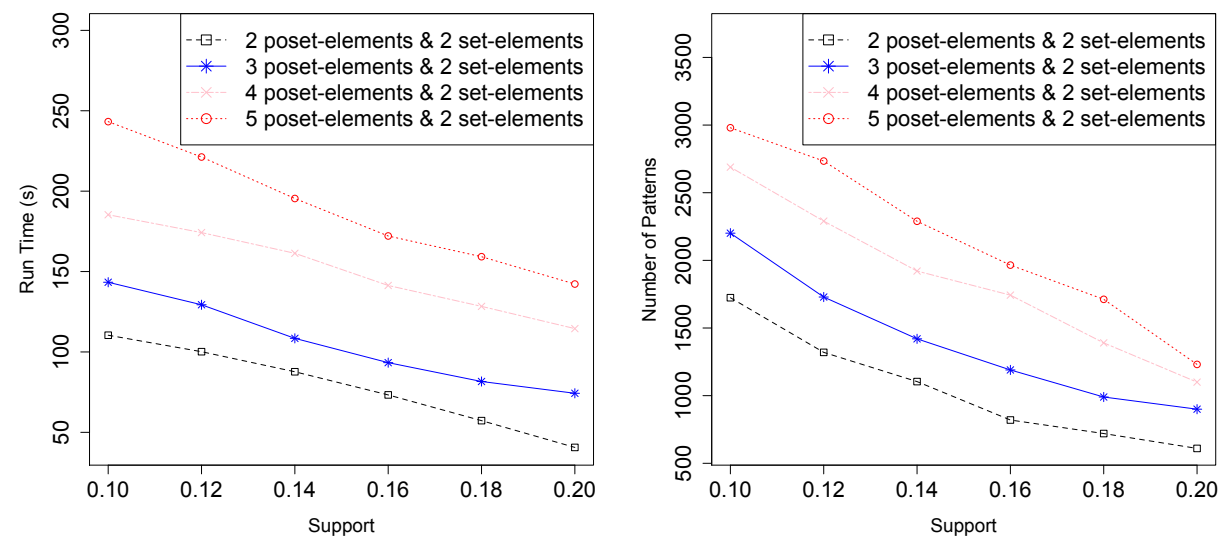

(a) Runtime sequences over frequency (b) Number patterns over frequency threshthreshold. old.

Fig. 9: Number of sequential pattern extracted and Running time obtained by MMISP with varying in the number of poset-elements.

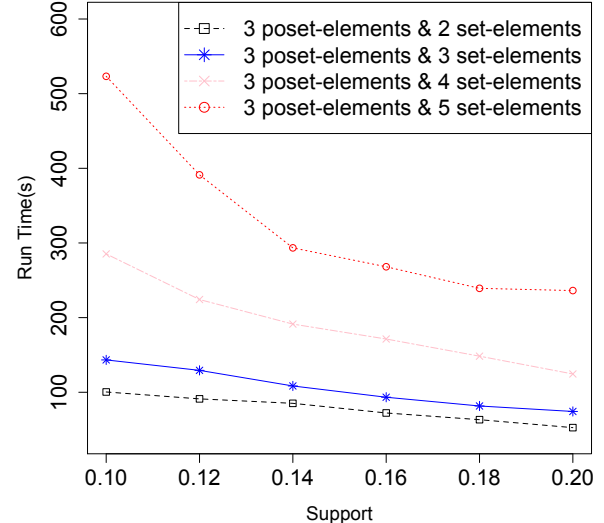

(a) Runtime sequences over frequency (b) Number patterns over frequency threshthreshold.

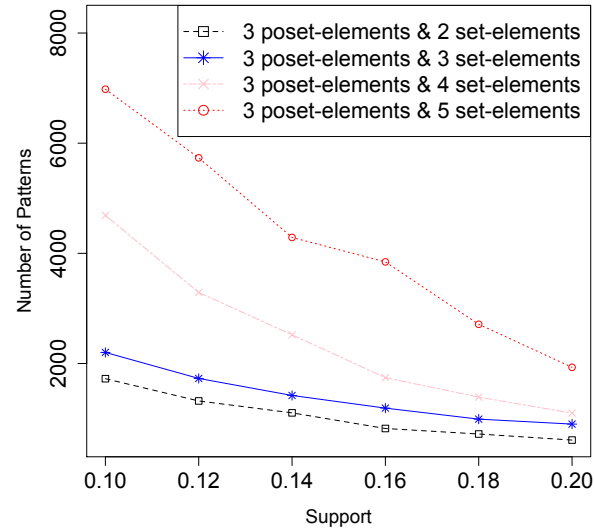

old.

Fig. 10: Number of sequential pattern extracted and Running time for a large panel of sequences when varying over the number of set-elements. 


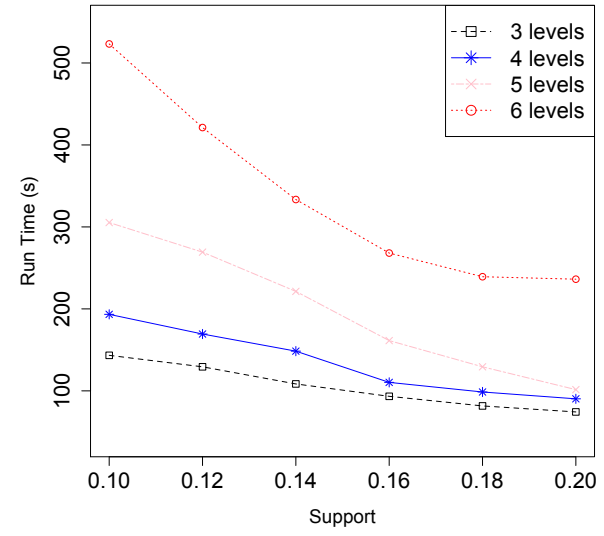

(a) Runtime sequences over frequency (b) Number patterns over frequency threshthreshold.

Fig. 11: Number of sequential pattern extracted and Running Time obtained for a large panel of sequences when varying over the levels of granularity between poset-elements.
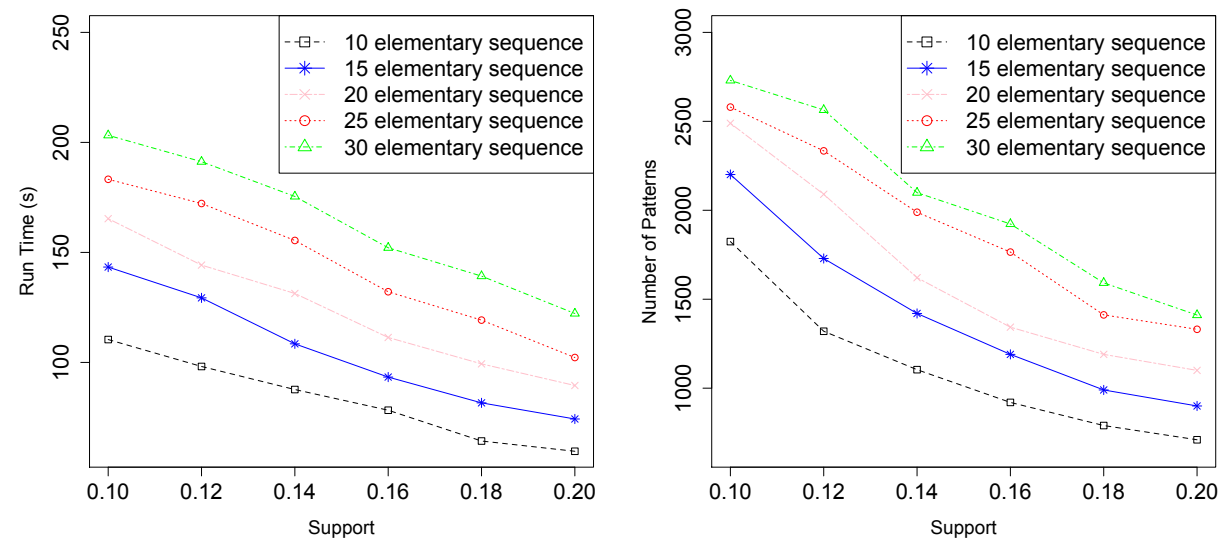

(a) Runtime sequences over frequency (b) Number patterns over frequency threshthreshold.

old.

Fig. 12: Number of sequential pattern extracted and Running time for a large panel of sequences when varying over sequence length. 


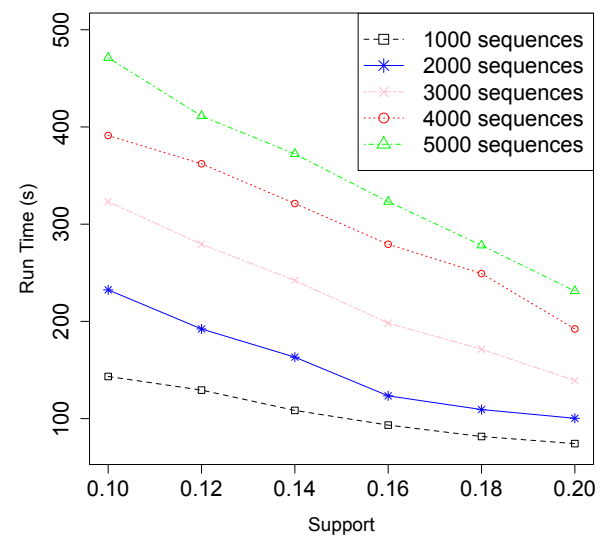

(a) Runtime sequences over frequency threshold.

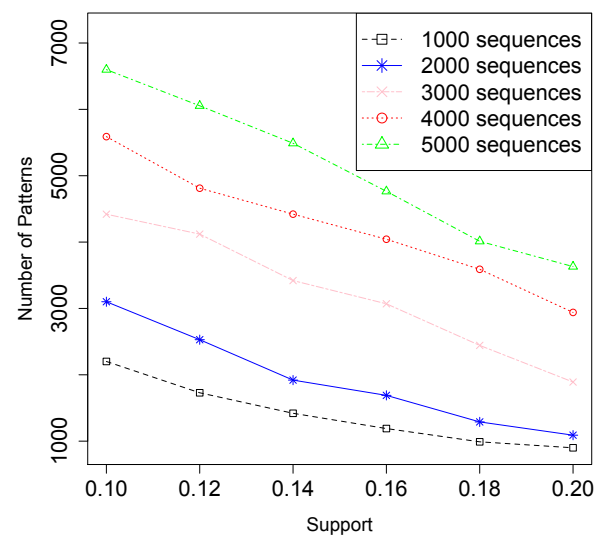

(b) Number patterns over frequency threshold.

Fig. 13: Number of sequential pattern extracted and Running time for a large panel of sequences when varying over several number of sequences.

\section{Conclusion}

In this paper, we propose a new approach to mine a sequential database of heterogeneous sequences. We provide formal definitions and propose a new algorithm MMISP to mine this kind of sequences. The MMISP algorithm relies on external posets to improve the mining process and produces results with appropriate levels of granularity. We conduct experiments on both real-world and synthetic datasets. The method is applied on real-world data where the problem is to mine healthcare patients trajectories and gives potential interesting patterns for healthcare specialists.

For future work, we are planning to use statistical significance tests to evaluate the sequential patterns extracted and choose the most significant ones. On the other hand, proposing a graphical interface to visualize and query the sequential patterns. We are also interested in generalizing our method by considering sequences all elements of which are lying in partially ordered set.

Finally, we are aware that choosing the most specific frequent elementary sequence to mine the sequential patterns prevents us from extracting all of the most specific sequential patterns. Coping with this issue is another interesting extension of the present work that we plan to investigate in the future. 


\section{Acknowledgments}

The authors would like to thank the French institution "Institut National du Cancer (INCa)" for its financial support.

\section{References}

1. Rakesh Agrawal and Ramakrishnan Srikant. Mining sequential patterns. In Proceedings of the Eleventh International Conference on Data Engineering, ICDE '95, pages 3-14, Washington, DC, USA, 1995. IEEE Computer Society.

2. Jay Ayres, Jason Flannick, Johannes Gehrke, and Tomi Yiu. Sequential pattern mining using a bitmap representation. In $K D D$, pages 429-435, 2002.

3. Ding-Ying Chiu, Yi-Hung $\mathrm{Wu}$, and Arbee L. P. Chen. An efficient algorithm for mining frequent sequences by a new strategy without support counting. In $I C D E$, pages 375-386, 2004.

4. RB Fetter, Y Shin, JL Freeman, RF Averill, and JD Thompson. Case mix definition by diagnosis-related groups. Med Care, 18(2):1-53, Feb 1980.

5. Florent Masseglia, Fabienne Cathala, and Pascal Poncelet. The PSP approach for mining sequential patterns. In PKDD, pages 176-184, 1998.

6. Carl H. Mooney and John F. Roddick. Sequential pattern mining - approaches and algorithms. ACM Comput. Surv., 45(2):19:1-19:39, March 2013.

7. Jian Pei, Jiawei Han, Behzad Mortazavi-Asl, Helen Pinto, Qiming Chen, Umeshwar Dayal, and Meichun Hsu. Prefixspan: Mining sequential patterns by prefixprojected growth. In ICDE, pages 215-224, 2001.

8. Helen Pinto, Jiawei Han, Jian Pei, Ke Wang, Qiming Chen, and Umeshwar Dayal. Multi-dimensional sequential pattern mining. In CIKM, pages 81-88, 2001.

9. Marc Plantevit, Anne Laurent, Dominique Laurent, Maguelonne Teisseire, and Yeow Wei Choong. Mining multidimensional and multilevel sequential patterns. TKDD, 4(1):1-37, 2010.

10. Eliana Salvemini, Fabio Fumarola, Donato Malerba, and Jiawei Han. Fast sequence mining based on sparse id-lists. In Proceedings of the 19th international conference on Foundations of intelligent systems, ISMIS'11, pages 316-325, Berlin, Heidelberg, 2011. Springer-Verlag.

11. Ramakrishnan Srikant and Rakesh Agrawal. Mining sequential patterns: Generalizations and performance improvements. In Proceedings of the 5th International Conference on Extending Database Technology: Advances in Database Technology, EDBT '96, pages 3-17, London, UK, UK, 1996. Springer-Verlag.

12. Xifeng Yan, Jiawei Han, and Ramin Afshar. Clospan: Mining closed sequential patterns in large datasets. In In SDM, pages 166-177, 2003.

13. Zhenglu Yang, Masaru Kitsuregawa, and Yitong Wang. Paid: Mining sequential patterns by passed item deduction in large databases. In IDEAS, pages 113-120, 2006.

14. Chung-Ching Yu and Yen-Liang Chen. Mining sequential patterns from multidimensional sequence data. IEEE Trans. Knowl. Data Eng., 17(1):136-140, 2005.

15. Cong $\mathrm{Yu}$ and H. V. Jagadish. Querying complex structured databases. In Proceedings of the 33rd international conference on Very large data bases, VLDB '07, pages 1010-1021. VLDB Endowment, 2007.

16. Mohammed J. Zaki. Spade: An efficient algorithm for mining frequent sequences. Mach. Learn., 42(1-2):31-60, January 2001. 
17. Changhai Zhang, Kongfa Hu, Zhuxi Chen, Ling Chen, and Yisheng Dong. Approxmgmsp: A scalable method of mining approximate multidimensional sequential patterns on distributed system. In FSKD (2), pages 730-734, 2007. 